Conference abstract PDD04

\title{
Stabilization of Human Growth Hormone Against Deamidation by Interaction with Strongly Basic Protamines from Salmon
}

\author{
E. AbLinger ${ }^{1,2}$, S. WegsCheider ${ }^{2}$, T. PAVkov-Keller ${ }^{3}$, \\ W. KELLER ${ }^{3}$, R. PrAssl ${ }^{4}$, A. ZIMMER ${ }^{2}$ \\ ${ }^{1}$ Research Center Pharmaceutical Engineering, Graz, Austria \\ ${ }^{2}$ Institute of Pharmaceutical Sciences, Department of Pharmaceutical Technology, University of Graz, Graz, \\ Austria \\ ${ }^{3}$ Institute of Molecular Biosciences. Structural Biology, University of Graz, Graz, Austria \\ ${ }^{4}$ Institute of Biophysics and Nanosystems Research, Austrian Academy of Sciences, Graz, Austria \\ E-mail: elisabeth.ablinger@tugraz.at (E. Ablinger)
}

Sci Pharm. 2010; 78: 593

doi:10.3797/scipharm.cespt.8.PDD04

Deamidation of proteins is probably one of the most common chemical degradation pathways in protein pharmaceuticals. The reaction rate for deamidation of human growth hormone $(\mathrm{hGH})$ is particularly $\mathrm{pH}$-dependent and occurs typically faster at neutral and alkaline $\mathrm{pH}$ [1].

Protamine from salmon is an extremely basic peptide due to its high arginine content. It is isolated from sperm cells of salmon where it naturally builds a complex with DNA during spermatogenesis due to electrostatic interactions [2]. We hypothesize a similar electrostatic effect between negatively charged hGH and positively charged protamine residues. Arising cationic complexes are stabilized by electrostatic repulsion. This stabilizing complexation allows solubilization of hGH down to a pH of 5.4 at high concentrations up to $6.8 \mathrm{mg} / \mathrm{ml}$. The minimal solubilizing molar ratio between $\mathrm{hGH}$ and protamine was found to be at least 1:13 (hGH:protamine). Deceleration of deamidation at pH 5.4 could be detected by reversed-phase high-performance liquid chromatography (RPHPLC). The presence of protamine did not alter the conformational structure of hGH which was determined by circular dichroism (CD) spectroscopy and fluorescence spectroscopy. Binding interactions between protamine and hGH were investigated by isothermal titration calorimetry (ITC) and Small-angle X-ray scattering (SAXS).

Acknowledgement: This work was supported by FFG, Land Steiermark and SFG.

[1] Manning MC, Patel K, Borchardt RT. Stability of Protein Pharmaceuticals. Pharm Res. 1989; 6: 903918. doi:10.1023/A:1015929109894

[2] Ando T, Yamasaki M, Suzuki K. Protamines-Isolation, Characterization, Structure and Function. Springer Verlag. 1973: 1-27. 